SMC were isolated via enzymatic dissociation of endarterectomy specimens and using immunomagnetic beads (Miltenyi). Commercially available aortic medial SMC from six healthy donors were purchased from Promocell. Cells were used consistently at passage 3. We used two-dimensional electrophoresis with digital image analysis (SameSpots, Non-linear Dynamics) and tandem mass spectrometry to detect changes in proteome of atherosclerotic SMC.

Results Analysis of 2D gel images revealed 29 proteins with a statistically significant difference in expression between medial and plaque SMC $(p<0.05)$. Plaque SMC had decreased expression of mitochondrial protein ATP synthase subunit $\beta$ but an increase in the oxidised form of peroxiredoxin- 4 , suggesting decreased mitochondrial function, possibly owing to oxidative stress. Glycolytic enzyme pyruvate kinase was also increased by $50 \%$ in plaque SMC. Furthermore, differences in protein expression between SMC from symptomatic and asymptomatic patients were also found. Plaque SMC from symptomatic patients exhibited increased expression of heat shock protein- 60 and decreased levels of the antiinflammatory protein annexin I $(p<0.05)$, compatible with a proinflammatory behaviour. These findings were confirmed by immunoblotting.

Conclusions Our data demonstrate that plaque-derived SMC are exposed to higher levels of oxidative stress than control SMC. Differences between SMC from symptomatic and asymptomatic patients appear to reflect proinflammatory changes associated with plaque instability.

\section{BAS/ INCREASED PLASMINOGEN ACTIVATOR INHIBITOR-1 MAY BSCR13 EXPLAIN DEXAMETHASONE-INDUCED THROMBOSIS AS SITE OF INTRALUMINAL WIRE INJURY}

doi:10.1136/hrt.2010.205781.24

L Low, J R Seckl, B R Walker, P W F Hadoke. Centre for Cardiovascular Science, The Queen's Medical Research Institute, University of Edinburgh, Scotland, UK

We have previously shown that high-dose glucocorticoid treatment reduces neointimal proliferation following arterial injury in mice, but is associated with increased local thrombosis. Clinically, glucocorticoid excess, either in Cushing's syndrome or with chronic treatment, is associated with increased coagulation and decreased fibrinolysis. The few animal (rat) studies which have investigated these effects support the conclusion that glucocorticoid treatment reduces fibrinolysis. This study aimed to determine the influence of glucocorticoid administration on the thrombotic potential in mice.

Male C57Bl/6J mice (aged 10-12 weeks) received either vehicle or dexamethasone (dex; 0.1 or $0.8 \mathrm{mg} / \mathrm{kg} /$ day) orally for 5 weeks $(\mathrm{n}=8 /$ group). Tail tip bleeding time was reduced by high-dose dex $(52.9 \pm 5.6 \mathrm{~s})$ compared with vehicle $(87.1 \pm 13.6 \mathrm{~s} ; \mathrm{p}<0.05)$. High-dose dex increased plasminogen activator inhibitor-1 (PAI-1; $139.5 \pm 15.1 \%$ vs vehicle $100.0 \pm 10.7 \% ; \mathrm{p}<0.05$ and decreased tissue plasminogen activator (tPA; $55.9 \pm 4.9 \%$ vs vehicle $100.0 \pm 10.3 \%$; $p<0.05$ ) mRNA levels in the heart. In addition, high-dose dex increased total PAI-1 $(3.53 \pm 0.59 \mathrm{ng} / \mathrm{ml}$ vs vehicle $0.96 \pm 0.17 \mathrm{ng} / \mathrm{ml} ; \mathrm{p}<0.001)$ and active PAI-1 $(0.92 \pm 0.09 \mathrm{ng} / \mathrm{ml}$ vs vehicle $0.31 \pm 0.07 \mathrm{ng} / \mathrm{ml} ; \mathrm{p}<0.001)$ plasma antigen levels. High-dose dex did not alter platelet activation as measured by p-selectin expression using flow cytometry. Low-dose dex had no effect on any parameters described.

Dexamethasone-induced thrombosis at the site of intraluminal wire injury may be attributable to alterations in the endogenous fibrinolytic system rather than changes in platelet activity. These results suggest that beneficial inhibition of neointimal proliferation mediated by systemic glucocorticoid administration is negated in part by changes in fibrinolysis leading to large, semi-occlusive thrombi formation at the site of injury.

\section{BAS/ LIPIDOMIC PROFILING OF HUMAN ATHEROSCLEROTIC BSCR14 PLAQUES}

doi:10.1136/hrt.2010.205781.25

${ }^{1} \mathrm{C}$ Stegemann, ${ }^{2} \mathrm{~J}$ Shalhoub, ${ }^{1} \mathrm{~J}$ Jenkins, ${ }^{3} \mathrm{~A} \mathrm{H}$ Davies, ${ }^{4} \mathrm{C}$ Ladroue, ${ }^{2} \mathrm{C}$ Monaco, ${ }^{1} \mathrm{~A}$ Smith, ${ }^{1} \mathrm{O} \mathrm{Xu},{ }^{1} \mathrm{M}$ Mayr. ${ }^{1} \mathrm{King}$ 's BHF Centre, King's College London, UK; ${ }^{2}$ Imperial College, London, UK; ${ }^{3}$ Imperial Vascular Unit, London, UK; ${ }^{4}$ University of Warwick, Warwick, England

Rationale Lipid accumulation in arteries is the hallmark of atherosclerosis. Yet, a comparative lipid analysis of human plaques using state-of-the-art mass spectrometry techniques has not been performed. Methods and results Comparative lipid profiling of radial arteries and carotid endarterectomies was performed by ultra performance liquid chromatography mass spectrometry. Lipid species were subsequently identified by shotgun lipidomics using a triple quadrupole mass spectrometer. Twenty-two different scan modes in positive and negative ion mode plus additional scans for different fatty acids resulted in the detection of 149 lipid species from nine different classes. Twenty-nine lipid species were only found in endarterectomy specimens. The main qualitative differences between control and diseased arteries were identified among lysophosphatidylcholines, sphingomyelins and cholesteryl esters (CE), including two oxidised CE species. Quantitative mass spectrometric analysis showed that the average concentration of CEs exceeded $30 \mathrm{pmol} / \mu \mathrm{l}$ in atherosclerotic plaques compared with only $0.3 \mathrm{pmol} / \mu \mathrm{l}$ in control arteries. Further analyses focused on plaques from symptomatic and asymptomatic patients and stable and unstable plaque areas from the same lesion. Principal component analysis confirmed that among all the lipids analysed most of the information was in the CEs but no clear discrimination could be obtained between patients $(n=24)$

Conclusions This study provides the most comprehensive lipid analysis of human atherosclerosis to date. Comparative lipid profiling of control and diseased arteries resulted in the identification of plaque-specific lipids, but failed to unambiguously identify vulnerable lesions.

\section{BAS/ THE ROLE OF PINK1, A MITOCHONDRIAL PRO-SURVIVAL BSCR15 KINASE, IN MYOCARDIAL ISCHAEMIA-REPERFUSION}

doi:10.1136/hrt.2010.205781.26

H Siddall, R Sands, S M Davidson, D J Hausenloy, M M Mocanu, D M Yellon. The Hatter Cardiovascular Institute, UCL Hospital and Medical School, London, UK

PINK1, a kinase localised mainly in mitochondria, can protect neurons against oxidative damage and apoptosis by maintaining mitochondrial structure and function. Although PINK1 is highly expressed in the myocardium, there are no studies investigating the role of this kinase in the heart. We hypothesised that PINK1 could protect cardiomyocytes from ischaemia-reperfusion (I-R) injury and therefore, PINK1 downregulation would be detrimental to the ischaemic-reperfused heart. Hearts isolated from PINK1+/+, PINK1 +/- and PINK1-/- mice were perfused in a Langendorff constant pressure system and subjected to $35 \mathrm{~min}$ global normothermic ischaemia and $30 \mathrm{~min}$ reperfusion. Infarct size was measured, using triphenyltetrazolium chloride (TTC) staining, and expressed as percentage of the myocardium at risk (I/R\%). Electron microscopy was used to investigate ultrastructural changes in cardiomyocytes, and the expression of autophagic markers such as Beclin1 and LC3b was measured in PINK1-/- hearts. Our data show that the PINK1 